Canad. Math. Bull. Vol. 21 (3), 1978

\title{
ON WEAK VITALI COVERING PROPERTIES
}

\author{
BY \\ B. S. THOMSON
}

There are now a number of Vitali covering properties which have been defined to handle problems arising in differentiation theory. Although some of these have received a unified treatment, as for example in the setting of Orlicz spaces in [1, p. 168], the underlying simplicity can be lost and the intimate connection with the original weak Vitali covering property of de Possel obscured. In this note we present an exposition of a family of covering properties and show how the original methods of de Possel in [4] can be pushed to provide an exact solution of the problem of determining necessary and sufficient covering properties for a basis which is known to differentiate a given class of integrals.

Throughout $(R, \mathfrak{M}, \mu)$ will denote a fixed measure space and $\mathfrak{M}_{0}$ and $\mathfrak{M}_{\mathrm{oo}}$ the classes of sets $\{M \in \mathfrak{M}: \mu(M)<+\infty\}$ and $\{M \in \mathfrak{M}: 0<\mu(M)<+\infty\}$ respectively. We assume that $\mu$ is complete in the sense that whenever $\mu(A)=0$ for every $A \subset B$ with $A \in \mathfrak{M}_{0}$ then necessarily $B \in \mathfrak{M}$ and $\mu(B)=0 . \mathfrak{B}$ is a derivation basis on $(R, \mathfrak{M}, \mu)$ so that for each $x \in R, \mathfrak{B}(x)$ is a filterbase of families of subsets from $\mathfrak{M}_{00}$. A class $\mathscr{V} \subset \mathfrak{M}_{00}$ is said to be a $\mathfrak{B}$-fine cover of a set $A \subset R$ if for every $x \in A$ and every $\mathscr{B} \in \mathfrak{B}(x), \mathscr{B} \cap \mathscr{V} \neq \varnothing$. We use both the expressions $\chi_{A}$ and $\chi(A)$ to denote the usual characteristic function of the set $A$ and whenever $\mathscr{E}$ is a sequence of sets $\left\{V_{1}, V_{2}, \cdots, V_{m}\right\}$ we use $\varphi_{\mathscr{E}}$ to denote the function

$$
t \rightarrow \varphi_{\mathscr{E}}(t)=\sum_{1}^{m} \chi\left(V_{i}, t\right)
$$

and $\sigma \mathscr{E}$ to denote the union of the sets in the sequence $\mathscr{E}$; always $\mathscr{E}$ will denote such a finite sequence of sets and our covering properties will be expressed in terms of approximation properties of such functions $\varphi_{\mathscr{E}}$ rather than directly in terms of covering/overlapping properties of the sets in the sequence itself.

A function which is integrable (finitely) on each set in the class $\mathfrak{M}_{0}$ is said to be locally integrable, and such expressions as $L_{p}$ (loc) are meant merely in this sense. For a locally integrable function $f$, the statement $\Phi=\int f d \mu$ indicates that $\Phi$ is a set function defined on $\mathfrak{M}_{0}$ by writing, for each $M \in \mathfrak{M}_{0}, \Phi(M)=\int_{M} f d \mu$. The upper derivates, the lower derivates, and the derivates $D^{*} \Phi, D_{*} \Phi$, and $D \Phi$ are defined at each point $x$ of $R$ in the obvious manner using the filterbase

Received by the editors November 14, 1977. 
$\mathfrak{B}(x) . \mathfrak{B}$ is said to derive a class $\mathscr{F}$ of locally integrable functions if almost everywhere in $R$

$$
D \Phi=f(x) \quad\left(\Phi=\int f d \mu\right)
$$

for each function $f \in \mathscr{F}$.

We now define a family of weak Vitali covering properties: let $\mathfrak{X}$ denote the linear space of all finite linear combinations of the functions $\chi_{M}$ for $M \in \mathfrak{M}_{0}$, and let $\tau$ represent any vector space topology on $\mathfrak{X}$.

Definition $1 . \mathfrak{B}$ is said to have the $\tau$-Vitali property if for every $\mathfrak{B}$-fine covering $\mathscr{V}$ of a set $A \in \mathfrak{M}_{0}$ the function $\chi_{\mathrm{A}}$ is in the $\tau$-closure of the set

$$
\left\{\varphi_{\mathscr{B}}: \mathscr{E} \subset \mathscr{V}\right\}
$$

where as usual $\mathscr{E}$ denotes an arbitrary finite sequence of sets from $\mathscr{V}$.

In most cases of interest $\mathfrak{X}$ is embedded in a larger topological vector space of integrable functions and $\tau$ is the appropriate subspace topology. For later reference and to clarify the ideas we present several examples:

(i) For any number $p, 1 \leq p<+\infty$, let $L_{p}$ denote the usual space of all $p$ th power integrable functions topologized by the seminorm

$$
\|f\|_{p}=\left(\int_{R}|f|^{p} d \mu\right)^{1 / p} .
$$

Then $\mathfrak{X}$ is a subspace of $L_{p}$ and we shall write $\tau_{p}$ as the induced topology on $\mathfrak{X}$. Note that the $\tau_{\mathrm{p}}$-Vitali property can be re-expressed as: for every $\mathfrak{B}$-fine covering $\mathscr{V}$ of a set $A \in \mathbb{M}_{0}$

$$
\inf \left\{\left\|\varphi_{\mathscr{B}}-\chi_{\mathrm{A}}\right\|_{p}: \mathscr{E} \subset \mathscr{V}\right\}=0 .
$$

(This property is related to the notion of an $S_{p}$-basis of Hayes and Pauc [1, p. 24].)

(ii) The particular case $p=1$ of the preceding is exactly the classical weak Vitali property of de Possel [4] and a basis with this property is called a weak derivation basis $[1$, p. 21$]$.

(iii) Let $\mathscr{F}$ be any collection of locally integrable functions and let $\sigma(\mathfrak{X}, \mathscr{F})$ denote the topology on $\mathfrak{X}$ generated by the family of seminorms (cf. [6, p. 48]) $\left\{N_{f}: f \in \mathscr{F}\right\}$ where

$$
N_{f}(g)=\left|\int_{R} f g d \mu\right|
$$

The standard problem arising in connection with such a family of covering properties is that of determining which properties guarantee that the basis derive a given class of locally integrable functions, and conversely, knowing that the basis does derive some such class of functions, to obtain covering 
properties which must then hold. The solution of the latter problem rests in general on the original work of de Possel [4]; although his methods were intended only for a basis known to derive the class $\left\{\chi_{M}: M \in \mathfrak{M}_{0}\right\}$ they in fact provide a complete formal solution to the problem. We summarize the essence of his contribution in a lemma.

Lemma (de Possel). Suppose that $\psi$ is a $\mu$-continuous measure on $\mathfrak{M}$, so that $0<\psi(M)<+\infty$ for every $M \in \mathfrak{M}_{\mathrm{oo}}$ and so that

$$
\lim _{W \Rightarrow x} \psi(M \cap W) / \psi(W)=1
$$

for at least one point $x$ of any set $M$ in $\mathfrak{M}_{\mathrm{oo}}$. Then for every positive number $\varepsilon$ and every $\mathfrak{B}$-fine covering $\mathscr{V}$ of a set $A \in \mathfrak{M}_{00}$ there exists a sequence $\mathscr{E} \subset \mathscr{V}$ with

$$
\int_{R}\left|\varphi_{\mathscr{8}}-\chi_{A}\right| d \psi<\varepsilon
$$

Proof. The proof uses the original inductive construction of [4] (cf. [1, pp. 30-31]) with a few modifications. Choose firstly a number $\delta, 0<\delta<1$, so that $2 \delta(1-\delta)^{-1} \psi(A)<\varepsilon$ and write $\mathscr{V}(Y, \varepsilon)$ for the collection of sets $V \in \mathscr{V}$ with

$$
|\psi(V)-\psi(Y \cap V)|<\delta \psi(V)
$$

or equivalently with

$$
\int_{R} \chi_{V}\left(1-\chi_{Y}\right) d \psi<\delta \psi(V)
$$

whenever $Y$ is some set in $\mathfrak{M}_{0}$. By our assumptions on $\psi$ and on $\mathscr{V}$ we may conclude that $\mathscr{V}(Y, \varepsilon) \neq \varnothing$ for any set $Y$ belonging to $\mathfrak{M}_{\mathrm{oo}}$ and contained in $A$; accordingly if we define

$$
v(Y)=\sup \{\psi(V): V \in \mathscr{V}(Y, \varepsilon)\}
$$

then $v(Y)$ is necessarily positive for any such $Y$, which is the key to the proof of assertion (ii) below.

We construct now a finite or infinite sequence of sets $\left\{V_{1}, V_{2}, V_{3}, \ldots\right\}$ from the class $\mathscr{V}$ by setting $X_{1}=A$ and choosing $V_{1} \in \mathscr{V}\left(X_{1}, \varepsilon\right)$ with $2 \psi\left(V_{1}\right)>v\left(X_{1}\right)$; and continuing inductively setting $X_{n+1}=A \backslash \bigcup_{1}^{n} V_{i}$ and choosing $V_{n+1} \epsilon$ $V\left(X_{n+1}, \varepsilon\right)$ with $2 \psi\left(V_{n+1}\right)>v\left(X_{n+1}\right)$. Note that the process continues as long as $v\left(X_{m}\right)$ is non-zero, terminating otherwise. We claim that the sequence $\left\{V_{1}, V_{2}, V_{3}, \ldots\right\}$ so constructed has the properties (i) $\sum \psi\left(V_{i}\right)<\psi(A)(1-\delta)^{-1}$ and (ii) $\psi\left(A \backslash \bigcup\left[V_{i} \cap X_{i}\right]\right)=0$.

To prove (i) note that each set $V_{i}$ belongs to $V\left(X_{i}, \varepsilon\right)$ so that $(1-\delta) \psi\left(V_{i}\right)<$ $\psi\left(V_{i} \cap X_{i}\right)$ and so, since the sets $\left\{X_{i} \cap V_{i}\right\}$ are by construction disjoint measurable subsets of $A$, we must have

$$
\sum(1-\delta) \psi\left(V_{i}\right)<\sum \psi\left(X_{i} \cap V_{i}\right)<\psi(A)
$$


which proves (i). For (ii) suppose on the contrary that $\psi(Z)>0$ where $Z=$ $A \backslash \bigcup\left[X_{i} \cap V_{i}\right]$. Then $Z \in \mathfrak{M}_{00}$ and $Z \subset A$ so that, as remarked above, $v(Z) \neq 0$ and from this we will derive a contradiction. For each $n$ we have $Z \subset X_{n}$ so that $\mathscr{V}(Z, \varepsilon) \subset \mathscr{V}\left(X_{n}, \varepsilon\right)$ and hence $v(Z) \leq v\left(X_{n}\right)$. However either $v\left(X_{m}\right)=0$ for some $m$ (in which case the sequence $\left\{V_{i}\right\}$ is finite) or else $\lim v\left(X_{n}\right)=0$ for we have by construction that $0 \leq v\left(X_{n}\right)<2 \psi\left(V_{n}\right)$ and we know by (i) that the series $\sum \psi\left(V_{n}\right)$ converges. In either case $v(Z)=0$ which is the desired contradiction and so (ii) follows.

Now define the finite sequence $\mathscr{E}=\left\{V_{1}, V_{2}, \ldots, V_{N}\right\}$ where the integer $N$ is chosen so that

$$
\int_{R}\left[\chi_{\mathrm{A}}-\sum_{1}^{N} \chi\left(V_{i}\right) \chi\left(X_{i}\right)\right] d \psi=\psi\left(A \backslash \bigcup_{1}^{N}\left[V_{i} \cap X_{i}\right]\right)<\varepsilon / 2
$$

which is possible because of (ii). By (i) and the choice of $\delta$ we have for any such choice of $N$ that

$$
\sum_{1}^{N} \int_{R}\left[\chi\left(V_{i}\right)-\chi\left(V_{i}\right) \chi\left(X_{i}\right)\right] d \psi<\delta \sum \psi\left(V_{i}\right)<\delta(1-\delta)^{-1} \psi(A)<\varepsilon / 2
$$

so that combining these two inequalities yields

$$
\int_{R}\left|\varphi_{\mathscr{E}}-\chi_{A}\right| d \psi \leq \int_{R}\left|\varphi_{\mathscr{B}}-\sum_{1}^{N} \chi\left(V_{i}\right) \chi\left(X_{i}\right)\right| d \psi+\int_{R}\left|\sum_{1}^{N} \chi\left(V_{i}\right) \chi\left(X_{i}\right)-\chi_{A}\right| d \psi<\varepsilon
$$

as required completing the proof.

We can now state the general covering/differentiation results. We depart only slightly from the usual conventions and separate the problem into two phases corresponding to the inequalities $D^{*} \Phi \leq f$ a.e. $\left(\Phi=\int f d \mu, f \geq 0\right.$ and $f$ in a given class $\mathscr{F})$ and $D_{*} \Phi \geq f$ a.e. $\left(\Phi=\int f d \mu, f \geq 0\right.$ and $f$ an arbitrary locally integrable function).

THEOREM 1. For every non-negative locally integrable function $f, D_{*} \Phi \geq f$ a.e. $\left(\Phi=\int f d \mu\right)$ if and only if the basis $\mathfrak{B}$ has the $\tau_{1}$-Vitali property.

Proof. Suppose that $\mathfrak{B}$ has the $\tau_{1}$-Vitali property and that $f$ and $\Phi$ are as stated in the theorem. Let $A_{\alpha \beta}$ for $0<\alpha<\beta$ denote the set of points $x$ in $R$ for which $D_{*} \Phi<\alpha<\beta<f(x)$, let $A$ be any measurable subset of $A_{\alpha \beta}$ with $A \in \mathfrak{M}_{0}$, and set $\mathscr{V}=\left\{M \in \mathfrak{M}_{00}: \Phi(M)<\alpha \mu(M)\right\}$. Clearly $\mathscr{V}$ is a $\mathfrak{B}$-fine covering of $\mathrm{A}$ and so by our assumptions on $\mathfrak{B}$ there is for any $\varepsilon>0$ a sequence $\mathscr{E} \subset \mathscr{V}$ with $\left\|\varphi_{\mathscr{E}}-\chi_{\mathrm{A}}\right\|_{1}$ so small that

$$
\max \left\{\Phi\left(A \backslash \sigma_{\mathscr{E}}^{\mathscr{E}}\right),\left\|\varphi_{\mathscr{E}}-\chi_{A}\right\|_{1}\right\}<\varepsilon
$$

(since $\Phi$ is an integral and $\left.\mu(A \backslash \sigma \mathscr{E}) \leq\left\|\varphi_{\mathscr{E}}-\chi_{A}\right\|_{1}\right)$. 
For such a sequence $\mathscr{E}$ a direct computation gives

$$
\begin{aligned}
\alpha^{-1}(\Phi(A)-\varepsilon) & \leq \alpha^{-1} \Phi(\sigma \mathscr{E}) \\
& \leq \alpha^{-1} \sum_{V \in \mathscr{Z}} \Phi(V) \\
& \leq \sum_{V \in \mathscr{Z}} \mu(V) \\
& =\int_{R} \varphi_{\mathscr{B}} d \mu \\
& =\int_{R}\left(\varphi_{\mathscr{B}}-\chi_{\mathbf{A}}\right) d \mu+\int_{R} \chi_{\mathbf{A}} d \mu \\
& \leq\left\|\varphi_{\mathscr{B}}-\chi_{\mathbf{A}}\right\|_{1}+\beta^{-1} \int_{A} f d \mu \\
& <\varepsilon+\beta^{-1} \Phi(A)
\end{aligned}
$$

so that $\alpha^{-1} \Phi(A) \leq \beta^{-1} \Phi(A)$ which is only possible if $\Phi(A)=0$; since we have $0 \leq \mu(A) \leq \beta^{-1} \Phi(A)$ we must also have $\mu(A)=0$ for any such $A \in \mathfrak{M}_{0}$. It follows then from our assumptions on $\mu$ that $\mu\left(A_{\alpha \beta}\right)=0$, and so finally by the standard argument of forming a union over all rational numbers $\alpha$ and $\beta$ we obtain

$$
\mu\left(\left\{x \in R: D_{*} \Phi<f(x)\right\}\right)=0
$$

as required.

The converse follows directly from the lemma by setting $\psi=\mu$.

Corollary. $\mathfrak{B}$ derives every function in $L_{\infty}(l o c)$ if and only if $\mathfrak{B}$ has the $\tau_{1}$-Vitali property.

Proof. The condition that $D_{*} \Phi \geq f$ a.e. for non-negative functions in $L_{1}$ (loc) is in fact equivalent to the statement that $\mathfrak{B}$ derives $L_{\infty}$ (loc).

It is well-known that the property of the theorem is not sufficient to guarantee that $\mathfrak{B}$ derives any particular unbounded function in $L_{1}$ (loc); in fact for the interval basis in the Euclidean plane, which does have the $\tau_{1}$-Vitali property with respect to Lebesgue measure, the celebrated theorem of S. Saks [5] shows that for "most" such functions (most in the category sense) $D^{*} \Phi=$ $+\infty$ everywhere. We obtain the result $D^{*} \Phi \leq f$ a.e. for all $f$ in some given class $\mathscr{F}$ of functions under some natural restrictions.

THEOREM 2. Let $\mathfrak{B}$ be a weak derivation basis and suppose that $\mathscr{F}$ is a class of locally integrable functions with $1 \in \mathscr{F}$ and $f \chi_{M} \in \mathscr{F}$ for every $f \in \mathscr{F}$ and every $M \in \mathfrak{M}_{00}$. Then $\mathfrak{B}$ derives $\mathscr{F}$ if and only if $\mathfrak{B}$ has the $\sigma(\mathfrak{X}, \mathscr{F})$-Vitali property. 
Proof. Under the hypotheses of the theorem to establish sufficiency we need only show that $D^{*} \Phi \leq f$ a.e. for every non-negative $f$ in $\mathscr{F}$. As in Theorem 1 for such an $f$ let $A_{\alpha \beta}$ denote the set of $x$ in $R$ for which $f(x)<\alpha<\beta<D^{*} \Phi$, let $A$ be any subset of $A_{\alpha \beta}$ belonging to $\mathfrak{M}_{0}$, and let $\mathscr{V}$ be the collection $\left\{M \in \mathfrak{M}_{\mathrm{oo}}: \Phi(M)>\beta \mu(M)\right\}$. Then $\mathscr{V}$ is a $\mathfrak{B}$-fine covering of $A$ and for every $\varepsilon>0$ we may select (by the $\sigma(\mathfrak{X}, \mathscr{F})$-Vitali property) a sequence $\mathscr{E} \subset \mathscr{V}$ with $N_{f}\left(\varphi_{\mathscr{B}}-\chi_{A}\right)<\varepsilon$ and $N_{1}\left(\varphi_{\mathscr{B}}-\chi_{A}\right)<\varepsilon$. A routine computation as before gives

$$
\begin{aligned}
\beta(\mu(A)-\varepsilon) & \leq \beta \sum_{V \in \mathscr{E}} \mu(V) \\
& \leq \sum_{V \in \mathscr{E}} \Phi(V) \\
& =\int_{R} \varphi_{\mathscr{E}} f d \mu \\
& =\int_{R}\left(\varphi_{\mathscr{E}}-\chi_{\mathrm{A}}\right) f d \mu+\int_{R} \chi_{A} f d \mu \\
& \leq N_{f}\left(\varphi_{\mathscr{E}}-\chi_{A}\right)+\alpha \mu(A)
\end{aligned}
$$

so that $\beta \mu(A) \leq \alpha \mu(A)$ which is only possible if $\mu(A)=0$ so that as before $\mu\left(A_{\alpha \beta}\right)=0$ and finally

$$
\mu\left(\left\{x \in R: D^{*} \Phi>f(x)\right\}=0\right.
$$

as required.

For the converse suppose that $\mathscr{V}$ is a $\mathfrak{B}$-fine covering of a set $A \in \mathfrak{M}_{0}$; we must show that every $\sigma(\mathfrak{X}, \mathscr{F})$-neighbourhood of $\chi_{\mathrm{A}}$ meets the set

$$
\left\{\varphi_{\mathscr{E}}: \mathscr{E} \subset \mathscr{V}\right\}
$$

For this take any finite collection $\left\{f_{1}, f_{2}, \ldots, f_{m}\right\}$ of non-negative functions from $\mathscr{F}$ : we establish the existence of a sequence $\mathscr{E} \subset \mathscr{V}$ such that

$$
\left|\int_{R}\left(\varphi_{\mathscr{E}}-\chi_{A}\right) f_{i} d \mu\right|<\varepsilon
$$

simultaneously for each $i=1,2, \ldots, m$. This is provided by the lemma with $\psi=\int\left(f_{1}+f_{2}+\cdots+f_{m}+1\right) d \mu$ and the theorem follows.

The hypotheses of the theorem are more natural than might be supposed at first glance: the requirement that $\mathfrak{B}$ derive all functions $\chi_{M}$ for $M \in \mathfrak{M}_{\mathrm{oo}}$ is equivalent to the statement that $\mathfrak{B}$ is a weak derivation basis, and for such a basis whenever $\mathfrak{B}$ derives a non-negative $f$ it necessarily derives $f_{\chi_{M}}$ (cf. $[1, \mathrm{p}$. 23]). It should be emphasized however that these theorems present only a formal solution to the covering/differentiation problem, a solution which is indeed implicit in the original de Possel equivalence theorem. There still remains the deeper problem of establishing interrelations between the various 
$\tau$-vitali covering properties: for example C. A. Hayes [2] has shown that the $\sigma\left(\mathfrak{X}, L_{q}\left(\right.\right.$ loc) ) - Vitali and the $\tau_{p}$-Vitali properties (where of course $p^{-1}+$ $q^{-1}=1$ ) are equivalent under very general conditions. A more delicate problem has been that of establishing the connection between the $\sigma\left(\mathfrak{X}, L_{\Psi}\right)$-Vitali and the $L_{\Phi}$-Vitali properties where $L_{\Phi}$ and $L_{\Psi}$ is an appropriate dual pair of Orlicz spaces; Hayes [3] has solved this problem too but has had to impose restrictions on the Orlicz functions $\Phi$ and $\Psi$ so that his results do not yet include every possible case of interest. It is hoped that our presentation, which places the problem directly in a geometrical and topological context, may lead to different techniques and further results.

\title{
REFERENCES
}

1. C. Hayes and C. Pauc, Derivation and Martingales, Springer-Verlag (1970).

2. C. Hayes, Derivation of integrals of $L^{(9)}$-functions, Pacific Jour. Math. 64 (1976), 173-180.

3. C. Hayes, Necessary and sufficient conditions for the derivation of integrals of $L_{\Psi^{-}}$-functions, Trans. Amer. Math. Soc. 223 (1976), 385-395.

4. R. de Possel, Dérivation abstraite des fonctions d'ensemble, Jour. de Math. Pures et Appl., 15 (1936), 391-409.

5. S. Saks, Remark on the differentiability of the Lebesgue indefinite integral, Fund. Math. 22 (1934), 257-261.

6. H. Schaeffer, Topological Vector Spaces, MacMillan (1966).

\author{
MATHEMATICS Department, \\ Simon Fraser UNIVERSITY \\ BURNABY, B.C. \\ Canada V5A 1 S6
}

